\title{
The Impact of Advances in Post-Mortem Imaging on Forensic Practice
}

\section{Bryce $\mathrm{CH}^{*}$}

\author{
Department of Pathology (Forensic Medicine), University of Edinburgh, United Kingdom
}

*Corresponding author: Bryce CH, Department of Pathology (Forensic Medicine), University of Edinburgh, Wilkie Building, Teviot Place, Edinburgh, EH8 9AG, United Kingdom, Tel: 0131650 2983, E-mail: clare. bryce@ed.ac.uk

\section{Citation: Bryce CH (2013) The Impact of Advances in Post-Mortem Imaging on Forensic Practice. J Forensic Sci Criminol 1(1): e103. doi: 10.15744/2348-9804.1.e103}

Received Date: July 01, 2013 Accepted Date: July 29, 2013 Published Date: August 01, 2013

Post-mortem imaging in the form of plain X-ray films has been in use for many years as an adjunct or occasionally as a substitute for autopsy. However, in the last two decades there has been increasing interest and investigation into the use of advanced techniques such as Computed Tomography (CT) and Magnetic Resonance Imaging (MRI) in death investigation.

Post-mortem CT imaging has several advantages over autopsy alone, since it produces high resolution detailed images of the body including areas that may not be easily accessible to the prosector, and allows documentation of abnormalities and injuries without the tissue disruption necessitated by the autopsy [1]. In addition, the image orientation (planes) and relative contrast of different tissues can be manipulated to better visualize different types of pathology. Three dimensional (3D) image construction is also possible with the appropriate software [1]. Several recent studies have compared the use of post mortem imaging with autopsy in injury evaluation. There was variable agreement between autopsy and CT findings, and CT was able to detect many injuries that were not found at autopsy [2]. One study showed that post mortem CT was more sensitive for detection of skeletal and head and neck region injuries compared with autopsy [3]. In this study, the authors used the Abbreviated Injury Scale, an anatomical-based coding system, to classify injuries in a standardized way and permit objective comparison between CT and autopsy. CT is also valuable in the documentation of gunshot wounds, not least because it allows three dimensional visualization of the wound course, as well as detection of subtle fractures and small bullet fragments that may be difficult to find at autopsy [1]. MRI and CT imaging has also been used in the investigation of child deaths, and CT is more sensitive for detection of rib fractures than conventional X-ray [1]. However, this is a relatively new use of the modality. One study involving 47 cases of sudden unexpected child deaths found a general concordance between autopsy and post mortem CT findings, except in a few cases of pneumonia [4].

A large validation study from the UK of 182 cases comparing whole body CT and MRI with full autopsy revealed a 32\% major discrepancy rate between radiology and autopsy findings. CT appeared to be a more accurate imaging technique than MRI for determination of cause of death. Common causes of sudden death were frequently missed by imaging; the most common errors being ischaemic heart disease, pulmonary embolism, pneumonia and intra-abdominal lesions. In general, CT provides better spatial resolution and demonstrates fractures and haemorrhages well, whereas MRI tends to provide better soft tissue detail [5].

Both CT and MRI are somewhat weak at identifying cardiac disease, including coronary artery lesions. Post-mortem CT angiography can be used to provide better visualization of blood vessels, with contrast medium being injected into the vascular system. However, this process takes some time (several hours) to perform [1]. A form of CT imaging called coronary optical coherence tomography has been used in the living to achieve such high resolution images of the coronary arteries that it has been termed minimally invasive virtual histology. Recently, this technique has been used for the first time in a post-mortem setting as part of a targeted CT angiography procedure to document extent of coronary artery disease [6].

As with any emerging technology, there are some limitations relating both to the procedure itself and its use post mortem. As previously mentioned, although CT and MRI are particularly useful in traumatic injury documentation, foreign body visualization and identifying gas collections, they are less accurate with some natural disease processes such as cardiac disease. These techniques also require some experience in distinguishing post mortem changes, such as internal gas production from decomposition, from true ante mortem pathology. In this setting, post mortem radiology can be considered a new sub-speciality requiring specific experience and training [7]. Indeed, most clinical radiologists will likely have little to no experience in interpretation of post mortem images and the injuries typically seen in a forensic setting. Similarly, most currently practicing forensic pathologists will have little or no experience in the interpretation of advanced imaging at autopsy. In some forensic facilities, particularly those affiliated with an academic centre; there is on-site CT and MRI, allowing close collaboration between forensic pathologists and radiologists with the possibility of "on the 
job" training. There is also scope for forensic imaging training to be incorporated into fellowship and continuing education programs [1].

Post mortem imaging has some distinct advantages. It can assist with providing a likely cause of death in cases where there is a strong objection to autopsy [5]. In some cases, it could be used with other minimally invasive procedures such as fine needle aspiration of a tumour mass, in order to come up with an accurate cause of death. Post mortem imaging, in particular 3D image construction, has potential value in the courtroom in that it would provide "bloodless" and easily understandable illustrations of injuries [1].

There are cost considerations in the use of these imaging modalities. CT is more widely available, less expensive and quicker to perform than MRI [5]. Another factor is the cost of maintenance of the equipment [1]. In many forensic departments, CT and MRI imaging is performed in the hospital radiology department. In this case, there are logistical considerations, such as agreeing a contract with the hospital radiology staff and working around the patient imaging schedule. In most cases, the post mortem imaging is done outof-hours, and so will require negotiations with staff regarding availability.

This is an exciting and rapidly developing area that is worthwhile for forensic practitioners to stay abreast of, since it is likely to impact their practice in some form in the future. Perhaps the best way to view these relatively new imaging techniques is as an adjunct to autopsy practice, rather than a replacement. In the first instance, the responsibility lies with the forensic pathologist as to how the technique will be incorporated into the work-up of an individual case, whether it will be combined with standard autopsy, or if it can be used in lieu of one.

\section{References}

1. Nolte KB, Mlady G, Zumwalt RE, Cushnyr B, Paul ID, et al. (2011) Postmortem X-ray Computed Tomography (CT) and Forensic Autopsy: A Review of the Utility, the Challenges and the Future Implications. Academic Forensic Pathology 1: 40-51.

2. Scholing M, Saltzherr TP, Fung Kon Jin PH, Ponsen KJ, Reitsma JB, et al. (2009) The value of postmortem computed tomography as an alternative for autopsy in trauma victims: a systematic review. Eur Radiol 19: 2333-41.

3. Daly B, Abboud S, Ali Z, Sliker C, Fowler D (2013) Comparison of wholebody post mortem 3D CT and autopsy evaluation in accidental blunt force traumatic death using the abbreviated injury scale classification. Forensic Sci Int 225: 20-6.

4. Proisy M, Marchand AJ, Loget P, Bouvet R, Roussey M, et al. (2013) Wholebody post-mortem computed tomography compared with autopsy in the investigation of unexpected death in infants and children. Eur Radiol 23: 171119.

5. Roberts IS, Benamore RE, Benbow EW, Lee SH, Harris JN, et al. (2012) Post-mortem imaging as an alternative to autopsy in the diagnosis of adult deaths: a validation study. Lancet 379: 136-42.

6. Adlam D, Joseph S, Robinson C, Rousseau C, Barber J, et al. (2013) Coronary optical coherence tomography: minimally invasive virtual histology as part of targeted post-mortem computed tomography angiography. Int J Legal Med 127: 991-6.

7. O'Donnell C, Woodford N (2008) Post-mortem radiology--a new subspeciality? Clin Radiol 63: 1189-94.

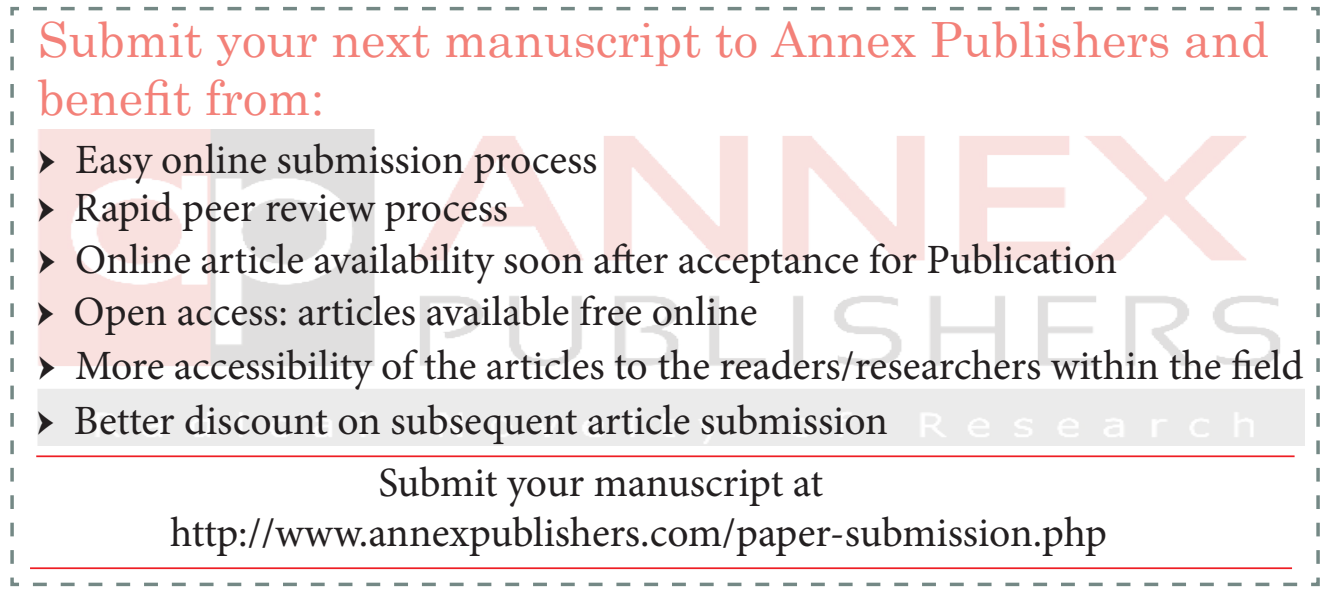

